\title{
FINITELY SUBADDITIVE OUTER MEASURES, FINITELY SUPERADDITIVE INNER MEASURES AND THEIR MEASURABLE SETS
}

\author{
P. D. STRATIGOS \\ Department of Mathematics \\ Long Island University \\ Brooklyn, NY 11201, USA
}

(Received February 24, 1994 and in revised form April 28, 1995)

\begin{abstract}
Consider any set $X$ A finitely subadditive outer measure on $\mathcal{P}(X)$ is defined to be a function $\nu$ from $\mathcal{P}(X)$ to $R$ such that $\nu(\emptyset)=0$ and $\nu$ is increasing and finitely subadditive A finitely superadditive inner measure on $\mathcal{P}(X)$ is defined to be a function $\rho$ from $\mathcal{P}(X)$ to $R$ such that $\rho(\emptyset)=0$ and $\rho$ is increasing and finitely superadditive (for disjoint unions) (It is to be noted that every finitely superadditive inner measure on $\mathcal{P}(X)$ is countably superadditive )

This paper contributes to the study of finitely subadditive outer measures on $\mathcal{P}(X)$ and finitely superadditive inner measures on $\mathcal{P}(X)$ and their measurable sets

KEY WORDS AND PHRASES. Lattice space, lattice normality and countable compactness, lattice regularity and $\sigma$-smoothness of a measure, finitely subadditive outer measure, finitely superadditive inner measure, regularity of a finitely subadditive outer measure
\end{abstract}

1991 AMS SUBJECT CLASSIFICATION CODES. 28A12, 28A60, 28C15

\section{INTRODUCTION}

Consider any set $X$ and any lattice on $X, \mathcal{L}$ The algebra on $X$ generated by $\mathcal{L}$ is denoted by $\mathcal{A}(\mathcal{L})$ A measure on $\mathcal{A}(\mathcal{L})$ is defined to be a function $\mu$ from $\mathcal{A}(\mathcal{L})$ to $R$ such that $\mu$ is finitely additive and bounded. The set of all measures on $\mathcal{A}(\mathcal{L})$ is denoted by $M(\mathcal{L})$ and the set of all $\mathcal{L}$-regular measures on $\mathcal{A}(\mathcal{L})$ by $M_{R}(\mathcal{L}) \quad$ A finitely subadditive outer measure on $\mathcal{P}(X)$ is defined to be a function $\nu$ from $\mathcal{P}(X)$ to $R$ such that $\nu(\emptyset)=0$ and $\nu$ is increasing and finitely subadditive A finitely superadditive inner measure on $\mathcal{P}(X)$ is defined to be a function $\rho$ from $\mathcal{P}(X)$ to $R$ such that $\rho(\emptyset)=0$ and $\rho$ is increasing and finitely superadditive (for disjoint unions). (It is to be noted that every finitely superadditive inner measure on $\mathcal{P}(X)$ is countably superadditive.) Associated with a measure $\mu$ on $\mathcal{A}(\mathcal{L})$, we introduce a variety of finitely subadditive outer measures on $\mathcal{P}(X)$ and finitely superadditive inner measures on $\mathcal{P}(X)$, and, in the case of $\mathcal{L}$ being normal, we utilize these set functions to give a short proof of the following fact ${ }^{*}$ For every measure $\mu$ on $\mathcal{A}(\mathcal{L})$ with $\mu \geq 0$, there exists an $\mathcal{L}$-regular measure $\nu$ on $\mathcal{A}(\mathcal{L})$ with $\nu \geq 0$, such that $\mu \leq \nu$ on $\mathcal{L}$ and $\mu(X)=\nu(X)$ and $\nu$ is unique (See Theorem 27 and the remark following it.)

These considerations lead us to a general analysis of finitely subadditive outer measures on $\mathcal{P}(X)$ and finitely superadditive inner measures on $\mathcal{P}(X) \quad$ [For a finite outer measure (countably subadditive) $\nu$ on $\mathcal{P}(X)$, it is well-known that the class of $\nu$-measurable sets - denoted by $\mathcal{S}_{\nu}$ - is a $\sigma$-algebra, that $\left.\nu\right|_{S_{\nu}}$ is a measure (countably additive), and that for every set $E, E \in \mathcal{S}_{\nu}$ iff $\nu^{0}(E)=\nu_{0}(E)$ where $\nu^{0}$ and $\nu_{0}$ are the outer measure and inner measure induced by $\left.\nu\right|_{\mathcal{S}_{\nu}}$, also, $\left.\mathcal{S}_{\nu^{0}}=\mathcal{S}_{\nu}=\mathcal{S}_{\nu_{0}}\right]$ The situation is vastly different in general for finitely subadditive outer measures, as we shall show. 
In general measure theory, it is shown that

(1) Every regular outer measure $\nu$ on $\mathcal{P}(X)$ has the following property For every sequence of sets $\left\langle E_{n}\right\rangle$, if $\left\langle E_{n}\right\rangle$ is increasing, then $\lim _{n} \nu\left(E_{n}\right)=\nu\left(\lim _{n} E_{n}\right)$

(2) Every finite regular outer measure $\nu$ on $\mathcal{P}(X)$ has the following property For every set $E$, $E \in \mathcal{S}_{\nu}$ if $\nu(E)+\nu\left(E^{\prime}\right)=\nu(X) \quad(*)$

We investigate these properties for finitely subadditive outer measures on $\mathcal{P}(X)$ The first property is false in general The second property remains true even though there are now two possibilities for defining regularity of $\nu$ In the course of our investigations, we also construct families of finitely subadditive outer measures on $\mathcal{P}(X)$ which are not necessarily regular but which still have Property (*) These are among the ones considered in the first part of the paper

Our terminology and notation are consistent with $[1,2,4,5]$ We review some terminology and notation and some basic facts for the reader's convenience Further matters can be found in $[3,4,8,9]$

\section{TERMINOLOGY AND NOTATION AND SOME BASIC FACTS}

(a) Consider any set $X$ and any lattice on $X, \mathcal{L}$ We shall assume that $\emptyset, X \in \mathcal{L}$, without loss of generality for our purposes

Now, consider any topological space $X$ and denote the class of open sets by $\mathcal{U}$ Note $\mathcal{U}$ is a lattice of the prescribed type $\operatorname{Recall} \mathcal{U}$ is referred to as the topology on $X$ and the topological space is defined to be $\langle X, \mathcal{U}\rangle$ Thus $\langle X, \mathcal{L}\rangle$ is a generalization of a topological space For this reason, we refer to $\langle X, \mathcal{L}\rangle$ as a lattice space

$\mathcal{L}$ is said to be normal iff for every two elements of $\mathcal{L}, A, B$, if $A \cap B=\emptyset$, then there exist two elements of $\mathcal{L}, C, D$, such that $A \subset C^{\prime}$ and $B \subset D^{\prime}$ and $C^{\prime} \cap D^{\prime}=\emptyset \quad \mathcal{L}$ is said to be countably compact iff for every sequence in $\mathcal{L},\left\langle L_{k}\right\rangle$, if $\cap_{k} L_{k}=\emptyset$, then there exists a value of $k, n$, such that $\cap_{k=1}^{n} L_{k}=\emptyset$

(b) The algebra on $X$ generated by $\mathcal{L}$ is denoted by $\mathcal{A}(\mathcal{L})$ Consider any algebra on $X, \mathcal{A}$ A measure on $\mathcal{A}$ is defined to be a function $\mu$ from $\mathcal{A}$ to $R$ such that $\mu$ is finitely additive and bounded (See [1], p 567.) The set whose general element is a measure on $\mathcal{A}(\mathcal{L})$ is denoted by $M(\mathcal{L})$ An element of $M(\mathcal{L}), \mu$, is said to be $\mathcal{L}$-regular iff for every element of $\mathcal{A}(\mathcal{L}), E$, for every positive number $\epsilon$, there exists an element of $\mathcal{L}, L$, such that $L \subset E$ and $|\mu(E)-\mu(L)|<\epsilon$ The set whose general element is an element of $M(\mathcal{L})$ which is $\mathcal{L}$-regular is denoted by $M_{R}(\mathcal{L})$ An element of $M(\mathcal{L}), \mu$, is said to be $\mathcal{L}$-( $\sigma$-smooth) iff for every sequence in $\mathcal{A}(\mathcal{L}),\left\langle A_{n}\right\rangle$, if $\left\langle A_{n}\right\rangle$ is decreasing and $\lim _{n} A_{n}=\emptyset$, then $\lim _{n} \mu\left(A_{n}\right)=0$. The set whose general element is an element of $M(\mathcal{L})$ which is $\mathcal{L}$ - $(\sigma$-smooth) is denoted by $M^{\sigma}(\mathcal{L})$. The set whose general element is an element of $M(\mathcal{L})$ which is $\mathcal{L}$-( $\sigma$-smooth $)$ just for $\left\langle A_{n}\right\rangle$ in $\mathcal{L}$ is denoted by $M_{\sigma}(\mathcal{L})$.

NOTE. Since every element of $M(\mathcal{L})$ is expressible as the difference of nonnegative elements of $M(\mathcal{L})$, we shall work with nonnegative elements of $M(\mathcal{L})$, without loss of generality

The set whose general element is an element of $M(\mathcal{L}), \mu$, such that $\mu(\mathcal{A}(\mathcal{L}))$ is $\{0,1\}$, that is, the set whose general element is a $(0-1)$-valued measure on $\mathcal{A}(\mathcal{L})$ is denoted by $I(\mathcal{L})$

(c) The following characterization of normality is known.

$\mathcal{L}$ is normal iff for every element of $I(\mathcal{L}), \mu$, for every two elements of $I_{R}(\mathcal{L}), \nu_{1}, \nu_{2}$, if $\mu \leq \nu_{1}, \nu_{2}$ on $\mathcal{L}$, then $\nu_{1}=\nu_{2}$.

Also, the following characterization of countable compactness is known.

$\mathcal{L}$ is countably compact iff $I_{R}(\mathcal{L}) \subset I_{R}^{o}(\mathcal{L})$.

\section{VARIOUS FINITELY SUBADDITIVE OUTER MEASURES AND FINITELY SUPERADDITIVE INNER MEASURES}

In this section, we deal with the following concepts submodular function, modular function, and supermodular function; finitely subadditive outer measure and finitely superadditive inner measure We give several examples. We study various properties of the outer and inner measures considered in our 
examples and we determine the relationship between them The concepts introduced in this section are also used in Section 3

Consider any set $X$ Now, consider any function $\nu$ from $\mathcal{P}(X)$ to $R$

DEFINITION 2.1. $\nu$ is submodular, modular, supermodular, iff for every two elements of $\mathcal{P}(X)$, $E_{1}, E_{2}, \nu\left(E_{1} \cup E_{2}\right)+\nu\left(E_{1} \cap E_{2}\right) \leq,(=),(\geq), \nu\left(E_{1}\right)+\nu\left(E_{2}\right)$, respectively

Observation If $\nu$ is modular, then $\nu$ is submodular and supermodular

DEFINITION 2.2. (a) $\nu$ is a finitely (countably) subadditive outer measure (on $\mathcal{P}(X)$ ) iff $\nu(\emptyset)=0$ and $\nu$ is increasing and finitely (countably) subadditive

(b) $\quad \nu$ is a finitely superadditive inner measure (on $\mathcal{P}(X)$ ) iff $\nu(\emptyset)=0$ and $\nu$ is increasing and finitely superadditive (for disjoint unions)

Observation If $\nu$ is a finitely superadditive inner measure, then $\nu$ is a countably superadditive inner measure

EXAMPLES. Consider any lattice space $\langle X, \mathcal{L}\rangle$ Now, consider any element of $M(\mathcal{L}), \mu$ (Note $\mu$ is modular )

A. Consider the function $\mu^{\prime}$ on $\mathcal{P}(X)$ determined by $\mu^{\prime}(E)=\inf \left\{\mu\left(L^{\prime}\right) \mid L^{\prime} \in \mathcal{L}^{\prime}\right.$ and $\left.L^{\prime} \supset E\right\}$

PROPOSITION 2.3. $\mu^{\prime}$ is submodular

(Proof omitted)

Observation $\mu^{\prime}$ is a finitely subadditive outer measure (This outer measure has been studied extensively in [9] )

B. Consider the function $\mu^{\prime \prime}$ on $\mathcal{P}(X)$ determined by $\mu^{\prime \prime}(E)=\inf \left\{\sum_{k=1}^{\infty} \mu\left(L_{k}^{\prime}\right) \mid\left\langle L_{k}^{\prime}\right\rangle\right.$ is in $\mathcal{L}^{\prime}$ and $\left.\cup_{k} L_{k}^{\prime} \supset E\right\}$

Note, $\mu^{\prime \prime}$ is a countably subadditive outer measure (This outer measure has been studied extensively in [9].)

C. Consider the function $\mu_{\imath}$ on $\mathcal{P}(X)$ determined by $\mu_{\imath}(E)=\sup \{\mu(L) \mid L \in \mathcal{L}$ and $L \subset E\}$

PROPOSITION 2.4. (1) $\mu_{\imath}$ is finite. (2) $\mu_{\imath}=\mu$ on $\mathcal{L}$. (3) $\mu_{\imath}$ is increasing (4) $\mu_{\imath}$ is supermodular (5) $\mu_{\imath}$ is a countably superadditive inner measure (on $\mathcal{P}(X)$ ). (6) If $\mathcal{L}$ is normal, then $\mu_{\imath}$ is finitely additive on $\mathcal{L}^{\prime}$.

PROOF. (4) Use the relevant definition. Consider any two elements of $\mathcal{P}(X), E_{1}, E_{2}$. Now, consider any positive number $\epsilon$. Denote any element of $\{1,2\}$ by $j$ Note for every $j$, since $\mu_{\imath}\left(E_{\jmath}\right)=\sup \left\{\mu(L) \mid L \in \mathcal{L}\right.$ and $\left.L \subset E_{\jmath}\right\}$ by the definition of $\mu_{2}$, and $\mu_{2}\left(E_{\jmath}\right)<+\infty$, there exists an element of $\mathcal{L}, L_{\jmath}$, such that $L_{\jmath} \subset E_{\jmath}$ and $\mu_{\imath}\left(L_{\jmath}\right)>\mu_{i}\left(E_{\jmath}\right)-\frac{\epsilon}{2}$, consider any such $L_{\jmath}$. Then $\mu_{\imath}\left(E_{1} \cup E_{2}\right) \geq \mu\left(L_{1} \cup L_{2}\right)$ and $\mu_{\imath}\left(E_{1} \cap E_{2}\right) \geq \mu\left(L_{1} \cap L_{2}\right)$, by the definition of $\mu_{\imath}$. Consequently $\mu_{\imath}\left(E_{1} \cup E_{2}\right)+\mu_{\imath}\left(E_{1} \cap E_{2}\right) \geq$ $\mu\left(L_{1} \cup L_{2}\right)+\mu\left(L_{1} \cap L_{2}\right)=\mu\left(L_{1}\right)+\mu\left(L_{2}\right)>\left(\mu_{i}\left(E_{1}\right)-\frac{\epsilon}{2}\right)+\left(\mu_{\imath}\left(E_{2}\right)-\frac{\epsilon}{2}\right)=\mu_{\imath}\left(E_{1}\right)+\mu_{2}\left(E_{2}\right)-\epsilon$ Hence $\mu_{\imath}\left(E_{1} \cup E_{2}\right)+\mu_{\imath}\left(E_{1} \cap E_{2}\right) \geq \mu_{\imath}\left(E_{1}\right)+\mu_{\imath}\left(E_{2}\right)$. Thus $\mu_{\imath}$ is supermodular.

(6) Assume $\mathcal{L}$ is normal. Consider any two elements of $\mathcal{L}^{\prime}, L_{1}^{\prime}, L_{2}^{\prime}$, such that $L_{1}^{\prime} \cap L_{2}^{\prime}=\emptyset$

$(\alpha)$ Note since $L_{1}^{\prime} \cap L_{2}^{\prime}=\emptyset, \mu_{\imath}\left(L_{1}^{\prime} \cup L_{2}^{\prime}\right) \geq \mu_{\imath}\left(L_{1}^{\prime}\right)+\mu_{\imath}\left(L_{2}^{\prime}\right)$, by (4)

( $\beta$ ) Show $\mu_{\imath}\left(L_{1}^{\prime} \cup L_{2}^{\prime}\right) \leq \mu_{\imath}\left(L_{1}^{\prime}\right)+\mu_{\imath}\left(L_{2}^{\prime}\right)$ Consider any element of $\mathcal{L}, A$, such that $A \subset L_{1}^{\prime} \cup L_{2}^{\prime}$ Then since $\mathcal{L}$ is normal by assumption, there exist two elements of $\mathcal{L}, B_{1}, B_{2}$ such that $A=B_{1} \cup B_{2}$ and $B_{1} \subset L_{1}^{\prime}$ and $B_{2} \subset L_{2}^{\prime}$. Consider any such $B_{1}, B_{2}$ Then

$$
\begin{aligned}
\mu(A)=\mu\left(B_{1} \cup B_{2}\right) & \leq \mu\left(B_{1}\right)+\mu\left(B_{2}\right) \\
& \leq \mu_{2}\left(L_{1}^{\prime}\right)+\mu_{2}\left(L_{2}^{\prime}\right),
\end{aligned}
$$

by the definition of $\mu_{2}$. Hence $\mu_{2}\left(L_{1}^{\prime} \cup L_{2}^{\prime}\right) \leq \mu_{2}\left(L_{1}^{\prime}\right)+\mu_{2}\left(L_{2}^{\prime}\right)$, by the definition of $\mu_{2}$

$(\gamma)$ Consequently $\mu_{\imath}\left(L_{1}^{\prime} \cup L_{2}^{\prime}\right)=\mu_{\imath}\left(L_{1}^{\prime}\right)+\mu_{\imath}\left(L_{2}^{\prime}\right)$. Thus $\mu_{\imath}$ is finitely additive on $\mathcal{L}^{\prime}$

D. Consider the function $\bar{\mu}$ on $\mathcal{P}(X)$ determined by $\bar{\mu}(E)=\inf \left\{\mu_{\imath}\left(L^{\prime}\right) \mid L^{\prime} \in \mathcal{L}^{\prime}\right.$ and $\left.L^{\prime} \supset E\right\}$ 
PROPOSITION 2.5. (1) $\bar{\mu}$ is finite $\quad$ (2) $\bar{\mu}=\mu_{2}$ on $\mathcal{L}^{\prime} \quad$ (3) $\mu_{\imath} \leq \bar{\mu}$ on $\mathcal{L} \quad$ (4) $\bar{\mu}$ is increasing

(Proof omitted)

PROPOSITION 2.6. (1) For every element of $\mathcal{P}(X), E, \mu_{\imath}(E)=\mu(X)-\mu^{\prime}\left(E^{\prime}\right) \quad$ (2) $\mu_{\imath} \leq \mu^{\prime}$ (3) $\mu_{\iota}=\mu^{\prime}$ on $\mathcal{L}$ iff $\mu \in M_{R}(\mathcal{L}) \quad$ (4) $\bar{\mu} \leq \mu^{\prime} \quad$ (5) $\bar{\mu}=\mu^{\prime}$ iff $\mu \in M_{R}(\mathcal{L}) \quad$ (6) If $\mathcal{L}$ is normal, then $\bar{\mu}=\mu^{\prime}$ on $\mathcal{L}$

PROOF. (5) Note for every element of $\mathcal{P}(X), E, \bar{\mu}(E)=\inf \left\{\mu_{\imath}\left(L^{\prime}\right) \mid L^{\prime} \in \mathcal{L}^{\prime}\right.$ and $\left.L^{\prime} \supset E\right\}$ by the definition of $\bar{\mu}$, and $\mu^{\prime}(E)=\inf \left\{\mu\left(L^{\prime}\right) \mid L^{\prime} \in \mathcal{L}^{\prime}\right.$ and $\left.L^{\prime} \supset E\right\}$ by the definition of $\mu^{\prime}$ Further, note $\bar{\mu}=\mu_{2}$ on $\mathcal{L}^{\prime}$ and $\mu^{\prime}=\mu$ on $\mathcal{L}^{\prime}$ Consequently $\bar{\mu}=\mu^{\prime}$ iff $\mu_{\imath}=\mu$ on $\mathcal{L}^{\prime}$ iff $\mu \in M_{R}(\mathcal{L})$

(6) Assume $\mathcal{L}$ is normal To show $\bar{\mu}=\mu^{\prime}$ on $\mathcal{L}$, assume the contrary Then since $\bar{\mu} \leq \mu^{\prime}$, there exists an element of $\mathcal{L}, A$, such that $\bar{\mu}(A)<\mu^{\prime}(A) \quad$ Consider any such $A$ Then since $\bar{\mu}(A)=\inf$ $\left\{\mu_{2}\left(L^{\prime}\right) \mid L^{\prime} \in \mathcal{L}^{\prime}\right.$ and $\left.L^{\prime} \supset A\right\}$ by the definition of $\bar{\mu}$, and $\bar{\mu}(A)<+\infty$, there exists an element of $\mathcal{L}^{\prime}$, $B^{\prime}$, such that $B^{\prime} \supset A$ and $\mu_{\imath}\left(B^{\prime}\right)<\mu^{\prime}(A)$ Consider any such $B^{\prime} \quad$ Then since $\mathcal{L}$ is normal by assumption, there exist two elements of $\mathcal{L}, C, D$, such that $A \subset C^{\prime} \subset D \subset B^{\prime}$ Consider any such $C$, $D$ Then $\mu\left(C^{\prime}\right) \leq \mu(D)=\mu_{\imath}(D) \leq \mu_{\imath}\left(B^{\prime}\right)<\mu^{\prime}(A)$ Thus $C^{\prime} \in \mathcal{L}^{\prime}$ and $C^{\prime} \supset A$ and $\mu\left(C^{\prime}\right)<\mu^{\prime}(A)$ Hence since $\mu^{\prime}(A)=\inf \left\{\mu\left(L^{\prime}\right) \mid L^{\prime} \in \mathcal{L}^{\prime}\right.$ and $\left.L^{\prime} \supset A\right\}$, this statement is false Therefore the assumption is wrong Consequently $\bar{\mu}=\mu^{\prime}$ on $\mathcal{L}$

THEOREM 2.7. If $\mathcal{L}$ is normal, then

(1) $\bar{\mu}$ is a finitely subadditive outer measure (on $\mathcal{P}(X)$ ).

Now, consider $\mathcal{S}_{\bar{\mu}}$ (the class of $\bar{\mu}$-measurable sets) Note $\mathcal{S}_{\bar{\mu}}$ is an algebra.

(2) $\mathcal{S}_{\bar{\mu}}=\left\{E \in \mathcal{P}(X) \mid\right.$ for every element of $\left.\mathcal{L}^{\prime}, A^{\prime}, \bar{\mu}\left(A^{\prime}\right) \geq \bar{\mu}\left(A^{\prime} \cap E\right)+\bar{\mu}\left(A^{\prime} \cap E^{\prime}\right)\right\}$

(3) (i) $\mathcal{L}^{\prime} \subset \mathcal{S}_{\bar{\mu}}$ (ii) $\mathcal{A}(\mathcal{L}) \subset \mathcal{S}_{\bar{\mu}}$.

Now, consider $\left.\bar{\mu}\right|_{\mathcal{A}(\mathcal{L})}$ Note $\left.\bar{\mu}\right|_{\mathcal{A}(\mathcal{L})} \in M(\mathcal{L})$ Denote it by $\nu$

(4) (i) $\nu \in M_{R}(\mathcal{L}) ; \mu \leq \nu$ on $\mathcal{L}$ and $\mu(X)=\nu(X)$. (ii) $\nu$ is unique.

PROOF. Assume $\mathcal{L}$ is normal

(1) Use the relevant definition. Note to show $\bar{\mu}$ is a finitely subadditive outer measure (on $\mathcal{P}(X)$ ), since $\bar{\mu}$ is finite and $\bar{\mu}(\emptyset)=0$ and $\bar{\mu}$ is increasing, it suffices to show $\bar{\mu}$ is finitely subadditive Accordingly, consider any two elements of $\mathcal{P}(X), E_{1}, E_{2}$. Now, consider any positive number $\epsilon$ Denote any element of $\{1,2\}$ by $j$. Note for every $j$, since $\bar{\mu}\left(E_{\jmath}\right)=\inf \left\{\mu_{\imath}\left(L^{\prime}\right) \mid L^{\prime} \in \mathcal{L}^{\prime}\right.$ and $\left.L^{\prime} \supset E_{\jmath}\right\}$ by the definition of $\bar{\mu}$, and $\bar{\mu}\left(E_{\jmath}\right)<+\infty$, there exists an element of $\mathcal{L}^{\prime}, L_{\jmath}^{\prime}$, such that $L_{\jmath}^{\prime} \supset E_{\jmath}$ and $\mu_{\imath}\left(L_{\jmath}^{\prime}\right)<\bar{\mu}\left(E_{\jmath}\right)+\frac{\epsilon}{2}$, consider any such $L_{\jmath}^{\prime}$. Then, $\mu_{\imath}\left(L_{1}^{\prime}\right)+\mu_{\imath}\left(L_{2}^{\prime}\right)<\bar{\mu}\left(E_{1}\right)+\bar{\mu}\left(E_{2}\right)+\epsilon$ Further, note since $\mathcal{L}$ is normal by assumption, $\mu_{\imath}\left(L_{1}^{\prime} \cup L_{2}^{\prime}\right) \leq \mu_{\imath}\left(L_{1}^{\prime}\right)+\mu_{\imath}\left(L_{2}^{\prime}\right)$, by (Prop. 2.4, (6)( $\beta$ )) Consequently $\mu_{2}\left(L_{1}^{\prime} \cup L_{2}^{\prime}\right) \leq \bar{\mu}\left(E_{1}\right)+\bar{\mu}\left(E_{2}\right)+\epsilon$. Then $\bar{\mu}\left(E_{1} \cup E_{2}\right)<\bar{\mu}\left(E_{1}\right)+\bar{\mu}\left(E_{2}\right)+\epsilon$, by the definition of $\bar{\mu}$. Hence $\bar{\mu}\left(E_{1} \cup E_{2}\right) \leq \bar{\mu}\left(E_{1}\right)+\bar{\mu}\left(E_{2}\right)$. Thus $\bar{\mu}$ is finitely subadditive Consequently $\bar{\mu}$ is a finitely subadditive outer measure (on $\mathcal{P}(X))$.

(2) Consider any element of $\mathcal{P}(X), E$.

$(\alpha)$ Assume $E \in \mathcal{S}_{\bar{\mu}}$. Then by the definition of $\bar{\mu}$-measurable set, for every element of $\mathcal{L}^{\prime}, A^{\prime}$, $\bar{\mu}\left(A^{\prime}\right) \geq \bar{\mu}\left(A^{\prime} \cap E\right)+\bar{\mu}\left(A^{\prime} \cap E^{\prime}\right)$.

( $\beta$ ) Assume for every element of $\mathcal{L}^{\prime}, A^{\prime}, \bar{\mu}\left(A^{\prime}\right) \geq \bar{\mu}\left(A^{\prime} \cap E\right)+\bar{\mu}\left(A^{\prime} \cap E^{\prime}\right)$. To show $\mathrm{E} \in \mathcal{S}_{\bar{\mu}}$, use the definition of $\bar{\mu}$-measurable set, namely, consider any element of $\mathcal{P}(X), G$, and show $\bar{\mu}(G) \geq \bar{\mu}(G \cap E)+\bar{\mu}\left(G \cap E^{\prime}\right)$. Note $\bar{\mu}(G)=\inf \left\{\mu_{\imath}\left(A^{\prime}\right) \mid A^{\prime} \in \mathcal{L}^{\prime}\right.$ and $\left.A^{\prime} \supset G\right\}$, by the definition of $\bar{\mu}$ Accordingly, consider any element of $\mathcal{L}^{\prime}, A^{\prime}$, such that $A^{\prime} \supset G$ Then

$$
\begin{aligned}
\mu_{\imath}\left(A^{\prime}\right) & =\bar{\mu}\left(A^{\prime}\right), \quad \text { since } \bar{\mu}=\mu_{2} \text { on } \mathcal{L}^{\prime}, \\
& \geq \bar{\mu}\left(A^{\prime} \cap E\right)+\bar{\mu}\left(A^{\prime} \cap E^{\prime}\right), \quad \text { by the assumption, } \\
& \geq \bar{\mu}(G \cap E)+\bar{\mu}\left(G \cap E^{\prime}\right), \quad \text { since } \bar{\mu} \text { is increasing }
\end{aligned}
$$

Consequently $\bar{\mu}(G) \geq \bar{\mu}(G \cap E)+\bar{\mu}\left(G \cap E^{\prime}\right)$. Thus $E \in \mathcal{S}_{\bar{\mu}}$. 
$(\gamma)$ Consequently $\mathcal{S}_{\mu}=\left\{E \in \mathcal{P}(X) \mid\right.$ for every element of $\mathcal{L}^{\prime}, \quad A^{\prime}, \quad \bar{\mu}\left(A^{\prime}\right) \geq \bar{\mu}\left(A^{\prime} \cap E\right)+$ $\left.\bar{\mu}\left(A^{\prime} \cap E^{\prime}\right)\right\}$

(3) (i) Consider any element of $\mathcal{L}^{\prime}, L^{\prime} \quad$ Note to show $L^{\prime} \in \mathcal{S}_{\bar{\mu}}$, according to (2), it suffices to show for every element of $\mathcal{L}^{\prime}, A^{\prime}, \bar{\mu}\left(A^{\prime}\right) \geq \bar{\mu}\left(A^{\prime} \cap L^{\prime}\right)+\bar{\mu}\left(A^{\prime} \cap L\right) \quad$ Accordingly, consider any element of $\mathcal{L}^{\prime}, A^{\prime}$ Then

$$
\begin{aligned}
\bar{\mu}\left(A^{\prime} \cap L^{\prime}\right) & =\mu_{\imath}\left(A^{\prime} \cap L^{\prime}\right), \quad \text { since } \bar{\mu}=\mu_{2} \quad \text { on } \mathcal{L}^{\prime} . \\
& =\sup \left\{\mu(\widehat{L}) \mid \widehat{L} \in \mathcal{L} \text { and } \widehat{L} \subset A^{\prime} \cap L^{\prime}\right\}, \text { by the definition of } \mu_{2}
\end{aligned}
$$

Now, consider any element of $\mathcal{L}, \widehat{L}$, such that $\widehat{L} \subset A^{\prime} \cap L^{\prime} \quad$ Further, note $\bar{\mu}\left(A^{\prime} \cap \widehat{L}^{\prime}\right)=\mu_{2}\left(A^{\prime} \cap \widehat{L}^{\prime}\right)=$ $\sup \left\{\mu(\tilde{L}) \mid \tilde{L} \in \mathcal{L}\right.$ and $\left.\tilde{L} \subset A^{\prime} \cap \widehat{L}^{\prime}\right\} \quad$ Now, consider any element of $\mathcal{L}, \tilde{L}$, such that $\tilde{L} \subset A^{\prime} \cap \widehat{L}^{\prime}$ Then

$$
\begin{aligned}
\bar{\mu}\left(A^{\prime}\right) & =\mu_{\imath}\left(A^{\prime}\right) \\
& \geq \mu(\widehat{L} \cup \tilde{L}), \quad \text { by the definition of } \mu_{\imath}, \\
& =\mu(\widehat{L})+\mu(\tilde{L}), \quad \text { since } \widehat{L} \cap \tilde{L}=\emptyset .
\end{aligned}
$$

Consequently $\bar{\mu}\left(A^{\prime}\right) \geq \mu(\widehat{L})+\bar{\mu}\left(A^{\prime} \cap \widehat{L}^{\prime}\right) \quad$ Then $\bar{\mu}\left(A^{\prime}\right) \geq \mu(\widehat{L})+\bar{\mu}\left(A^{\prime} \cap L\right)$, since $\widehat{L}^{\prime} \supset L \quad$ Consequently $\bar{\mu}\left(A^{\prime}\right) \geq \bar{\mu}\left(A^{\prime} \cap L^{\prime}\right)+\bar{\mu}\left(A^{\prime} \cap L\right) \quad$ Thus $L^{\prime} \in \mathcal{S}_{\bar{\mu}}$ Hence $\mathcal{L}^{\prime} \subset \mathcal{S}_{\bar{\mu}}$

(4) (i) Note for every element of $\mathcal{L}, A, \nu(A)=\bar{\mu}(A)=\inf \left\{\mu_{\imath}\left(L^{\prime}\right) \mid L^{\prime} \in \mathcal{L}^{\prime}\right.$ and $\left.L^{\prime} \supset A\right\}=$ $\inf \left\{\bar{\mu}\left(L^{\prime}\right) \mid L^{\prime} \in \mathcal{L}^{\prime}\right.$ and $\left.L^{\prime} \supset A\right\}=\inf \left\{\nu\left(L^{\prime}\right) \mid L^{\prime} \in \mathcal{L}^{\prime}\right.$ and $\left.L^{\prime} \supset A\right\} \quad$ Hence $\nu$ is $\mathcal{L}$-regular Consequently $\nu \in M_{R}(\mathcal{L}) \quad$ Further, note for every element of $\mathcal{L}, L, \mu(L)=\mu_{i}(L) \leq \bar{\mu}(L)=\nu(L)$ Hence $\mu \leq \nu$ on $\mathcal{L}$ Also, note $\mu(X)=\mu_{\imath}(X)=\bar{\mu}(X)=\nu(X)$

(ii) Consider any two elements of $M_{R}(\mathcal{L}), \nu_{1}, \nu_{2}$, such that $\mu \leq \nu_{1}, \nu_{2}$ on $\mathcal{L}$. Then since $\mathcal{L}$ is normal by assumption, $\mu^{\prime}=\nu_{1}^{\prime}, \nu_{2}^{\prime}$ on $\mathcal{L}$. (See [10] ) Further, note since $\nu_{1}, \nu_{2} \in M_{R}(\mathcal{L}), \nu_{1}=\nu_{1}^{\prime}$ and $\nu_{2}=\nu_{2}^{\prime}$ Consequently $\nu_{1}=\nu_{2}$ Thus $\nu$ is unique.

REMARK. We note that if $\mathcal{L}$ is not normal, then $\bar{\mu}$ need not be a finitely subadditive outer measure However, it has been shown that for every element of $M(\mathcal{L}), \mu$, there exists an element of $M_{R}(\mathcal{L}), \nu$, such that $\mu \leq \nu$ on $\mathcal{L}$ and $\mu(X)=\nu(X)$, although in general $\nu$ is not unique Different type proofs of this fact have been given in $[11,7,6]$. Some of these proofs use tools from functional analysis, others use Zorn's Lemma Our proof is measure theoretical and "constructive" and it also settles the question of uniqueness.

COROLLARY 2.8. If $\mathcal{L}$ is normal, then $\bar{\mu}$ is submodular

PROOF. Assume $\mathcal{L}$ is normal. Now, use the relevant definition Consider any two elements of $\mathcal{P}(X), E_{1}, E_{2}$. Now, consider any positive number $\epsilon$ Denote any element of $\{1,2\}$ by $j$. Then for every $j$, since $\bar{\mu}\left(E_{\jmath}\right)=\inf \left\{\mu_{2}\left(L^{\prime}\right) \mid L^{\prime} \in \mathcal{L}^{\prime}\right.$ and $\left.L^{\prime} \supset E_{\jmath}\right\}$ by the definition of $\bar{\mu}$, and $\bar{\mu}\left(E_{\jmath}\right)<+\infty$, there exists an element of $\mathcal{L}^{\prime}, L_{\jmath}^{\prime}$, such that $L_{\jmath}^{\prime} \supset E_{\jmath}$ and $\mu_{\imath}\left(L_{\jmath}^{\prime}\right)<\bar{\mu}\left(E_{\jmath}\right)+\frac{\epsilon}{2}$; consider any such $L_{\jmath}^{\prime}$ Then $\bar{\mu}\left(E_{1} \cup E_{2}\right) \leq \mu_{2}\left(L_{1}^{\prime} \cup L_{2}^{\prime}\right)$ and $\bar{\mu}\left(E_{1} \cap E_{2}\right) \leq \mu_{2}\left(L_{1}^{\prime} \cap L_{2}^{\prime}\right)$, by the definition of $\bar{\mu}$. Consequently $\bar{\mu}\left(E_{1} \cup E_{2}\right)+\bar{\mu}\left(E_{1} \cap E_{2}\right) \leq \mu_{2}\left(L_{1}^{\prime} \cup L_{2}^{\prime}\right)+\mu_{2}\left(L_{1}^{\prime} \cap L_{2}^{\prime}\right)=\bar{\mu}\left(L_{1}^{\prime} \cup L_{2}^{\prime}\right)+\bar{\mu}\left(L_{1}^{\prime} \cap L_{2}^{\prime}\right)$. Now, recall since $\mathcal{L}$ is normal by assumption, $\bar{\mu}$ is a finitely subadditive outer measure and $\mathcal{A}(\mathcal{L}) \subset \mathcal{S}_{\bar{\mu}} \quad$ (See Thm. 2.7) Then since $\left.\bar{\mu}\right|_{\mathcal{A}(\mathcal{L})}$ is a measure, $\bar{\mu}\left(L_{1}^{\prime} \cup L_{2}^{\prime}\right)+\bar{\mu}\left(L_{1}^{\prime} \cap L_{2}^{\prime}\right)=\bar{\mu}\left(L_{1}^{\prime}\right)+\bar{\mu}\left(L_{2}^{\prime}\right) \quad$ Consequently $\bar{\mu}\left(E_{1} \cup E_{2}\right)+\bar{\mu}\left(E_{1} \cap E_{2}\right) \leq \bar{\mu}\left(L_{1}^{\prime} \cup L_{2}^{\prime}\right)+\bar{\mu}\left(L_{1}^{\prime} \cap L_{2}^{\prime}\right)=\bar{\mu}\left(L_{1}^{\prime}\right)+\bar{\mu}\left(L_{2}^{\prime}\right)=\mu_{2}\left(L_{1}^{\prime}\right)+\mu_{2}\left(L_{2}^{\prime}\right)$ $<\left(\bar{\mu}\left(E_{1}\right)+\frac{\epsilon}{2}\right)+\left(\bar{\mu}\left(E_{2}\right)+\frac{\epsilon}{2}\right)=\bar{\mu}\left(E_{1}\right)+\bar{\mu}\left(E_{2}\right)+\epsilon \quad$ Hence $\bar{\mu}\left(E_{1} \cup E_{2}\right)+\bar{\mu}\left(E_{1} \cap E_{2}\right) \leq \bar{\mu}\left(E_{1}\right)+$ $\bar{\mu}\left(E_{2}\right)$ Thus $\bar{\mu}$ is submodular

PROPOSITION 2.9. If for every element of $I(\mathcal{L}), \mu, \bar{\mu}$ is submodular, then $\mathcal{L}$ is normal.

PROOF. To show $\mathcal{L}$ is normal, assume the contrary. Then according to the theory (see INTRODUCTION, (c)), there exist an element of $I(\mathcal{L}), \mu$, and two elements of $I_{R}(\mathcal{L}), \nu_{1}, \nu_{2}$, such that $\mu \leq \nu_{1}, \nu_{2}$ on $\mathcal{L}$ and $\nu_{1} \neq \nu_{2}$. Consider any such $\mu, \nu_{1}, \nu_{2}$ Then since $\nu_{1}, \nu_{2} \in I_{R}(\mathcal{L})$, there exist two elements of $\mathcal{L}, A_{1}, A_{2}$, such that $A_{1} \cap A_{2}=\emptyset ; \nu_{1}\left(A_{1}\right)=1$ and $\nu_{1}\left(A_{2}\right)=0 ; \nu_{2}\left(A_{1}\right)=0$ and $\nu_{2}\left(A_{2}\right)=1$. Consider any such $A_{1}, A_{2}$ Now, denote any element of $\{1,2\}$ by $j$ Then for every 
ग, $\mu^{\prime}\left(A_{\jmath}\right)=\inf \left\{\mu\left(L^{\prime}\right) \mid L^{\prime} \in \mathcal{L}^{\prime}\right.$ and $\left.L^{\prime} \supset A_{\jmath}\right\}$, by the definition of $\mu^{\prime}$, hence since $\nu_{\jmath}\left(A_{\jmath}\right)=1$, and $\mu, \nu_{\jmath} \in I(\mathcal{L})$ and $\mu \leq \nu_{\jmath}$ on $\mathcal{L}, \mu^{\prime}\left(A_{\jmath}\right)=1$ Further, note since $A_{1} \cap A_{2}=\emptyset, \mu^{\prime}\left(A_{1} \cap A_{2}\right)=0$ Next, note since $\mu_{\imath}=\bar{\mu}$ on $\mathcal{L}^{\prime}$ and $\bar{\mu}$ is submodular by assumption, $\mu_{2}$ is submodular on $\mathcal{L}^{\prime}$ Hence since for every element of $\mathcal{P}(X), E, \mu^{\prime}(E)=\nu(X)-\mu_{2}\left(E^{\prime}\right), \mu^{\prime}$ is supermodular on $\mathcal{L}$. Consequently $\mu^{\prime}\left(A_{1}\right)+\mu^{\prime}\left(A_{2}\right) \leq \mu^{\prime}\left(A_{1} \cup A_{2}\right)+\mu^{\prime}\left(A_{1} \cap A_{2}\right) \quad$ Hence since $\mu^{\prime}\left(A_{1}\right)=1, \mu^{\prime}\left(A_{2}\right)=1$, and $\mu^{\prime}\left(A_{1} \cap A_{2}\right)=0,1+1 \leq \mu^{\prime}\left(A_{1} \cup A_{2}\right)+0 \leq 1$ Therefore the assumption is wrong Consequently $\mathcal{L}$ is normal

Corollary 28 and Proposition 29 dictate the following

THEOREM 2.10. $\mathcal{L}$ is normal iff for every element of $I(\mathcal{L}), \mu, \bar{\mu}$ is submodular

\section{SET-MEASURABILITY AND PROPERTY (*)}

In [8] the following fact is established

Every finite regular outer measure $\nu$ has the following property

For every subset of $X, E$, if $\nu(E)+\nu\left(E^{\prime}\right)=\nu(X)$, then $E$ is $\nu$-measurable (*) (p. 96, Thm 122 )

In [8] an example is also given of a finite outer measure which although not regular, has Property (*) (p 95, Ex. g)

In this section, we investigate the importance of Property $(*)$ without regard to regularity. More specifically

(a) With an arbitrary finitely subadditive outer measure $\nu$ (on $\mathcal{P}(X)$ ) which is submodular, we associate a certain countably superadditive inner measure $\rho$ (on $\mathcal{P}(X)$ ), we determine the class of $\rho$ measurable sets, we also determine the relationship between the classes of $\nu$-measurable sets and $\rho$ measurable sets, using Property $(*)$ during the process.

(b) With an arbitrary finitely subadditive outer measure $\nu$ (on $\mathcal{P}(X)$ ), we associate a certain finitely subadditive outer measure $\nu^{0}$ (on $\mathcal{P}(X)$ ) and a certain countably superadditive inner measure $\nu_{0}$ (on $\mathcal{P}(X)$ ) using the class of $\nu$-measurable sets, (incidentally, we define regularity of $\nu$ in terms of $\nu^{0}$ ) we determine the relationship between $\nu, \nu^{0}$, and $\nu_{0}$; we also determine the relationship between the classes of $\nu$-measurable, $\nu^{0}$-measurable, and $\nu_{0}$-measurable sets, using Property $(*)$ during the process.

(c) We consider two arbitrary finitely subadditive outer measures $\nu_{1}, \nu_{2}$ (on $\mathcal{P}(X)$ ) such that $\nu_{1} \leq \nu_{2}$ and $\nu_{1}(X)=\nu_{2}(X)$; we determine the relationship between the associated classes of measurable sets and the relationship between $\nu_{\jmath 0}$ and $\nu_{j}^{0}$ for $j=1,2$, using Property (*) during the process. We give several examples.

Consider any finitely subadditive outer measure $\nu$ (on $\mathcal{P}(X)$ ). Now, consider the function $\rho$ on $\mathcal{P}(X)$ determined by $\rho(E)=\nu(X)-\nu\left(E^{\prime}\right)$.

\section{Part (a)}

PROPOSITION 3.1. $\rho$ is supermodular iff $\nu$ is submodular.

(Proof omitted.)

COROLLARY 3.2. If $\nu$ is submodular, then $\rho$ is a countably superadditive inner measure.

(Proof omitted.)

PROPOSITION 3.3. If $\nu$ is submodular, then
(1) $\mathcal{S}_{\rho}=\{E \in \mathcal{P}(X) \mid \rho(E)=\nu(E)\}$.
(2) $\mathcal{S}_{\nu} \subset \mathcal{S}_{\rho}$
(3) $\mathcal{S}_{\nu}=\mathcal{S}_{\rho}$ iff $\nu$ has Property (*).

PROOF. Assume $\nu$ is submodular.

(1) ( $\alpha$ ) Show $\mathcal{S}_{\rho} \subset\{E \in \mathcal{P}(X) \mid \rho(E)=\nu(E)\}$. Consider any element of $\mathcal{S}_{\rho}, E$. Then $\rho(X)=\rho(E)+\rho\left(E^{\prime}\right)$, by the definition of $\rho$-measurable set. Also, $\nu(X)=\nu(E)+\rho\left(E^{\prime}\right)$, by the definition of $\rho$. Consequently $\rho(E)+\rho\left(E^{\prime}\right)=\nu(E)+\rho\left(E^{\prime}\right)$. Hence since $\rho\left(E^{\prime}\right)<+\infty$, $\rho(E)=\nu(E)$. Thus $\mathcal{S}_{\rho} \subset\{E \in \mathcal{P}(X) \mid \rho(E)=\nu(E)\}$. 
( $\beta$ ) Show $\{E \in \mathcal{P}(X) \mid \rho(E)=\nu(E)\} \subset \mathcal{S}_{\rho} \quad$ Consider any element of $\mathcal{P}(X), E$, such that $\rho(E)=\nu(E)$ Note to show $E \in \mathcal{S}_{\rho}$, since $\rho$ is a finitely superadditive inner measure (on $\mathcal{P}(X)$ ) (see Cor 32 ), it suffices to show for every element of $\mathcal{P}(X), G, \rho(G) \leq \rho(G \cap E)+\rho\left(G \cap E^{\prime}\right)$ Accordingly, consider any element of $\mathcal{P}(X), G$ Note since $\nu$ is submodular by assumption, $\rho$ is supermodular (See Prop 31 ) Then

$$
\rho(G \cap E)+\rho\left(G \cap E^{\prime}\right) \geq(\rho(G)+\rho(E)-\rho(G \cup E))+\left(\rho(G)+\rho\left(E^{\prime}\right)-\rho\left(G \cup E^{\prime}\right)\right)
$$

(since $\rho$ is supermodular and $\rho$ is finite)

$$
=2 \rho(G)+\rho(X)-\left(\rho(G \cup E)+\rho\left(G \cup E^{\prime}\right)\right)
$$

(using the definition of $\rho$ and the assumption $\rho(E)=\nu(E)$ )

$$
\geq 2 \rho(G)+\rho(X)-\left(\rho\left((G \cup E) \cup\left(G \cup E^{\prime}\right)\right)+\rho\left((G \cup E) \cap\left(G \cup E^{\prime}\right)\right)\right)
$$

(since $\rho$ is supermodular)

$$
=2 \rho(G)+\rho(X)-(\rho(X)+\rho(G))=\rho(G) .
$$

Hence $\rho(G) \leq \rho(G \cap E)+\rho\left(G \cap E^{\prime}\right)$ Consequently $E \in \mathcal{S}_{\rho} \quad$ Thus $\{E \in \mathcal{P}(X) \mid \rho(E)=\nu(E)\} \subset \mathcal{S}_{\rho}$

( $\gamma$ ) Consequently $\mathcal{S}_{\rho}=\{E \in \mathcal{P}(X) \mid \rho(E)=\nu(E)\}$

(2) Consider any element of $\mathcal{S}_{\nu}, E$ Then $\nu(X)=\nu(E)+\nu\left(E^{\prime}\right)$, by the definition of $\nu$ measurable set Hence $\nu(E)=\nu(X)-\nu\left(E^{\prime}\right)$. Consequently $\rho(E)=\nu(E)$. Then $E \in \mathcal{S}_{\rho} \quad$ Thus $\mathcal{S}_{\nu} \subset \mathcal{S}_{\rho}$

(3) ( $\alpha$ ) Assume $\mathcal{S}_{\nu}=\mathcal{S}_{\rho}$ and show $\nu$ has Property (*) Consider any element of $\mathcal{P}(X), E$, such that $\nu(X)=\nu(E)+\nu\left(E^{\prime}\right)$ and show $E \in \mathcal{S}_{\nu}$. Note $\nu(X)=\rho(E)+\nu\left(E^{\prime}\right)$, by the definition of $\rho$ Consequently $\rho(E)=\nu(E) \quad$ Then $E \in \mathcal{S}_{\rho}$, by (1). Hence since $\mathcal{S}_{\rho} \subset \mathcal{S}_{\nu}$ by assumption, $E \in \mathcal{S}_{\nu}$ Consequently $\nu$ has Property $(*)$

( $\beta$ ) Assume $\nu$ has Property (*) and show $\mathcal{S}_{\nu}=\mathcal{S}_{\rho}$ Note for this, since $\mathcal{S}_{\nu} \subset \mathcal{S}_{\rho}$ by (2), it suffices to show $\mathcal{S}_{\rho} \subset \mathcal{S}_{\nu}$ Consider any element of $\mathcal{S}_{\rho}, E$. Note to show $E \in \mathcal{S}_{\nu}$, since $\nu$ has Property (*), it suffices to show $\nu(X)=\nu(E)+\nu\left(E^{\prime}\right) \quad$ Note since $E \in \mathcal{S}_{\rho}, \rho(E)=\nu(E)$, by (1) Hence since $\nu(X)=\rho(E)+\nu\left(E^{\prime}\right)$ by the definition of $\rho, \nu(X)=\nu(E)+\nu\left(E^{\prime}\right) \quad$ Consequently $E \in \mathcal{S}_{\nu} \quad$ Thus $\mathcal{S}_{\rho} \subset \mathcal{S}_{\nu}$. Consequently $\mathcal{S}_{\nu}=\mathcal{S}_{\rho}$.

\section{Part (b)}

Recall the facts: $\mathcal{S}_{\nu}$ is an algebra and $\left.\nu\right|_{\nu}$ is a measure

(a) Consider the function $\nu^{0}$ on $\mathcal{P}(X)$ determined by $\nu^{0}(E)=\inf \left\{\nu(S) \mid S \in \mathcal{S}_{\nu}\right.$ and $\left.S \supset E\right\}$.

$(\beta)$ Consider the function $\nu^{0}$ on $\mathcal{P}(X)$ determined by $\nu_{0}(E)=\sup \left\{\nu(S) \mid S \in \mathcal{S}_{\nu}\right.$ and $\left.S \subset E\right\}$

PROPOSITION 3.4. (1) $\nu^{0}$ is submodular (2) $\nu_{0}$ is supermodular.

(Proof omitted.)

PROPOSITION 3.5. (1) $\nu_{0} \leq \nu \leq \nu^{0}$ and $\nu_{0}=\nu=\nu^{0}$ on $\mathcal{S}_{\nu}$.

(2) (i) $\nu^{0}$ is a finitely subadditive outer measure (on $\mathcal{P}(X)$ ).

(ii) $\nu_{0}$ is a countably superadditive inner measure (on $\mathcal{P}(X)$ )

(3) For every element of $\mathcal{S}_{\nu}, E$, for every element of $\mathcal{P}(X), G$, if $G \subset E$, then $\nu_{0}(G)=$ $\nu(E)-\nu^{0}(E-G)$.

(4) For every element of $\mathcal{P}(X), G, \nu_{0}(G)+\nu^{0}\left(G^{\prime}\right)=\nu(X)$

(5) For every element of $\mathcal{S}_{\nu}, E, \nu(E)+\nu\left(E^{\prime}\right)=\nu(X)$.

PROOF. (2) (ii) Note the function $\nu_{0}$ is from $\mathcal{P}(X)$ to $R, \nu_{0}(\emptyset)=0$, and $\nu_{0}$ is increasing Further, note since $\nu_{0}$ is supermodular (see Prop 3.4, (ii)), $\nu_{0}$ is finitely superadditive Consequently $\nu_{0}$ is a countably superadditive inner measure (on $\mathcal{P}(X)$ ) 
(3) Consider any element of $\mathcal{S}_{\nu}, E$, and any element of $\mathcal{P}(X), G$, such that $G \subset E$ Note to show $\nu_{0}(G)=\nu(E)-\nu^{0}(E-G)$, according to the definition of $\nu_{0}$, it suffices to show $\nu(E)-\nu^{0}(E-G)=\sup \left\{\nu(S) \mid S \in \mathcal{S}_{\nu}\right.$ and $\left.S \subset G\right\}$

( $\alpha$ ) Show $\nu(E)-\nu^{0}(E-G)$ is an upper bound of $\left\{\nu(S) \mid S \in \mathcal{S}_{\nu}\right.$ and $\left.S \subset G\right\}$ Accordingly, consider any element of $\mathcal{S}_{\nu}, S$, such that $S \subset G$ and show $\nu(S) \leq \nu(E)-\nu^{0}(E-G)$ Note $\nu(S) \leq \nu(E)-\nu^{0}(E-G)$ iff $\quad \nu(E)-\nu(S) \geq \nu^{0}(E-G) \quad$ Now, consider $E-S$ and note $E-S \in \mathcal{S}_{\nu}$, since $E, S \in \mathcal{S}_{\nu}$ and $E-S \supset E-G$, since $S \subset G$ Hence by the definition of $\nu^{0}, \nu(E-S) \geq \nu^{0}(E-G) \quad$ Further, note since $\left.\nu\right|_{S_{\nu}}$ is a measure and $S \subset G \subset E, \nu(E-S)=$ $\nu(E)-\nu(S)$ Consequently $\nu(E)-\nu(S) \geq \nu^{0}(E-G) \quad$ Hence $\nu(S) \leq \nu(E)-\nu^{0}(E-G) \quad$ Thus $\nu(E)-\nu^{0}(E-G)$ is an upper bound of $\left\{\nu(S) \mid S \in \mathcal{S}_{\nu}\right.$ and $\left.S \subset G\right\}$

( $\beta$ ) Show for every positive number $\epsilon$, there exists an element of $\mathcal{S}_{\nu}, S$, such that $S \subset G$ and $\nu(S)>\left(\nu(E)-\nu^{0}(E-G)\right)-\epsilon \quad$ Accordingly, consider any positive number $\epsilon$ Then since $\nu^{0}(E-G)=\inf \left\{\nu(S) \mid S \in \mathcal{S}_{\nu}\right.$ and $\left.S \supset E-G\right\}$ by the definition of $\nu^{0}$, and $\nu^{0}(E-G)<+\infty$, there exists an element of $\mathcal{S}_{\nu}, S$, such that $S \supset E-G$ and $\nu(S)<\nu^{0}(E-G)+\epsilon$ Consider any such $S$ Then $-\nu(S)>-\nu^{0}(E-G)-\epsilon \quad$ Hence $\nu(E)-\nu(S)>\left(\nu(E)-\nu^{0}(E-G)\right)-\epsilon \quad$ Hence $\nu(E)-\nu(E \cap S)>\left(\nu(E)-\nu^{0}(E-G)\right)-\epsilon$ Consequently $\left.\nu(E-E \cap S)>\nu(E)-\nu^{0}(E-G)\right)-\epsilon$ Thus $E-E \cap S \in \mathcal{S}_{\nu}$ and $E-E \cap S \subset G$ and $\nu(E-E \cap S)>\left(\nu(E)-\nu^{0}(E-G)\right)-\epsilon$

$(\gamma)$ Consequently $\nu_{0}(G)=\nu(E)-\nu^{0}(E-G)$.

The following proposition will dictate the definition of regularity of a finitely subadditive outer measure $\nu$ (on $\mathcal{P}(X)$ )

PROPOSITION 3.6. Consider the following statements.

For every element of $\mathcal{P}(X), E$, there exists an element of $\mathcal{S}_{\nu}, S$, such that $S \supset E$ and

$$
\begin{gathered}
\nu(S)=\nu(E) . \\
\nu=\nu^{0} .
\end{gathered}
$$

Then (1) implies (2)

PROOF. Assume (1) Consider any element of $\mathcal{P}(X), E$. Then since (1) is true, there exists an element of $\mathcal{S}_{\nu}, S$, such that $S \supset E$ and $\nu(S)=\nu(E)$. Consider any such $S$. Now, recall $\nu^{0}(E)=\inf$ $\left\{\nu(N) \mid N \in \mathcal{S}_{\nu}\right.$ and $\left.N \supset E\right\}$, by the definition of $\nu^{0} \quad$ Consequently $\nu(E) \leq \nu^{0}(E) \leq \nu(S)=\nu(E)$ Hence $\nu(E)=\nu^{0}(E)$. Thus $\nu=\nu^{0}$, i.e., (2) is true

Observation If $\nu$ is countably subadditive, then (2) implies (1)

DEFINITION 3.7. $\nu$ is regular iff $\nu=\nu^{0}$.

In [8] the following fact is established:

Every regular outer measure $\mu^{*}$ has the following property:

For every increasing sequence of sets, $\left\langle A_{n}\right\rangle, \mu^{*}\left(\lim _{n} A_{n}\right)=\lim _{n} \mu^{*}\left(A_{n}\right)$. (\#) (p. 95, Cor. 12.11.)

To show that the countable subadditivity of $\mu^{*}$ is essential in the proof of this fact, we will present a finitely subadditive outer measure which although regular, does not have Property (\#), namely:

Consider any lattice space $\langle X, \mathcal{L}\rangle$ such that $\mathcal{L}$ is not countably compact. Then according to the theory (see INTRODUCTION, (c)), $I(\mathcal{L}) \not \subset I_{\sigma}(\mathcal{L})$. Hence there exists an element of $I(\mathcal{L}), \mu$, such that $\mu \notin I_{\sigma}(\mathcal{L}) \quad$ Consider any such $\mu$ Note $\mu^{\prime}$ is a finitely subadditive outer measure (on $\mathcal{P}(X)$ ) which is regular. Further, note since $\mu \notin I_{\sigma}(\mathcal{L})$, there exists a sequence in $\mathcal{L}^{\prime},\left\langle L_{n}^{\prime}\right\rangle$, such that $\left\langle L_{n}^{\prime}\right\rangle$ is increasing and $\lim _{n} L_{n}^{\prime}=X$ and for every $n, \mu\left(L_{n}^{\prime}\right)=0$ Consider any such $\left\langle L_{n}^{\prime}\right\rangle$ Note $\mu^{\prime}\left(\lim _{n} L_{n}^{\prime}\right)=\mu^{\prime}(X)=1$ and $\lim _{n} \mu^{\prime}\left(L_{n}^{\prime}\right)=0$. Hence $\mu^{\prime}\left(\lim _{n} L_{n}^{\prime}\right) \neq \lim _{n} \mu^{\prime}\left(L_{n}^{\prime}\right)$. Thus $\mu^{\prime}$ does not have Property (\#).

Observation. In general, every finitely subaditive outer measure $\nu$ on $\mathcal{P}(X)$, that is not countably subadditive, does not have Property (\#) (because, if it did, it would be countably subadditive).

Consider the function $\rho$ defined in Part (a). 
PROPOSITION 3.8. $\nu$ is regular iff $\rho=\nu_{0}$

PROOF. Note $\nu$ is regular iff $\nu=\nu^{0}$ (by definition) iff for every element of $\mathcal{P}(X) E$, $\nu(E)=\nu^{0}(E)=\inf \left\{\nu(S) \mid S \in \mathcal{S}_{\nu}\right.$ and $\left.S \supset E\right\}$ (by the definition of $\nu^{0}$ ) iff $\nu(X)-\nu(E)=\sup$ $\left\{\nu\left(S^{\prime}\right) \mid S^{\prime} \in \mathcal{S}_{\nu}^{\prime}\right.$ and $\left.S^{\prime} \subset E^{\prime}\right\}$ (since $\nu(X)<+\infty$ and $\left.\nu\right|_{\mathcal{S}_{\nu}}$ is a measure) iff $\rho\left(E^{\prime}\right)=\nu_{0}\left(E^{\prime}\right)$ (by the definitions of $\rho$ and $\left.\nu_{0}\right)$ iff $\rho=\nu_{0}$

PROPOSITION 3.9. If $\nu$ is regular, then $\nu$ has Property (*)

PROOF. (Slight modification of proof in [8] for countably subadditive outer measures )

Assume $\nu$ is regular To show $\nu$ has Property $(*)$, consider any element of $\mathcal{P}(X), E$, such that $\nu(E)+\nu\left(E^{\prime}\right)=\nu(X)$ and show $E \in \mathcal{S}_{\nu}$ Note to show $E \in \mathcal{S}_{\nu}$, it suffices to show for every element of $\mathcal{P}(X), G, \nu(G \cap E)+\nu\left(G \cap E^{\prime}\right) \leq \nu(G)$ Accordingly, consider any element of $\mathcal{P}(X), G$ Now, consider any positive number $\epsilon$ Then since $\nu^{0}(G)=\inf \left\{\nu(N) \mid N \in \mathcal{S}_{\nu}\right.$ and $\left.N \supset G\right\}$ and $\nu^{0}(G)<+\infty$, there exists an element of $\mathcal{S}_{\nu}, N$, such that $N \supset G$ and $\nu(N)<\nu^{0}(G)+\epsilon$ Consider any such $N$ Then

$$
\begin{aligned}
\nu(X) & =\nu(E)+\nu\left(E^{\prime}\right), \quad \text { by assumption, } \\
& =\left(\nu(E \cap N)+\nu\left(E \cap N^{\prime}\right)\right)+\left(\nu\left(E^{\prime} \cap N\right)+\nu\left(E^{\prime} \cap N^{\prime}\right)\right), \quad \text { since } \quad N \in \mathcal{S}_{\nu}, \\
& =\left(\nu(E \cap N)+\nu\left(E^{\prime} \cap N\right)\right)+\left(\nu\left(E \cap N^{\prime}\right)+\nu\left(E^{\prime} \cap N^{\prime}\right)\right) \\
& \geq \nu(N)+\nu\left(N^{\prime}\right), \quad \text { since } \nu \text { is finitely subadditive, } \\
& =\nu(X), \quad \text { since } \quad N \in \mathcal{S}_{\nu} .
\end{aligned}
$$

Hence $\quad\left(\nu(E \cap N)+\nu\left(E^{\prime} \cap N\right)\right)+\left(\nu\left(E \cap N^{\prime}\right)+\nu\left(E^{\prime} \cap N^{\prime}\right)\right)=\nu(N)+\nu\left(N^{\prime}\right) \quad$ Hence since $\nu\left(E \cap N^{\prime}\right)+\nu\left(E^{\prime} \cap N^{\prime}\right) \geq \nu\left(N^{\prime}\right), \nu(E \cap N)+\nu\left(E^{\prime} \cap N\right) \leq \nu(N) \quad$ Consequently $\quad \nu(G \cap E)+$ $\nu\left(G \cap E^{\prime}\right) \leq \nu(N \cap E)+\nu\left(N \cap E^{\prime}\right) \leq \nu(N)<\nu^{0}(G)+\epsilon \quad$ Now, note since $\nu$ is regular by assumption, $\nu=\nu^{0}$ by definition Consequently $\nu(G \cap E)+\nu\left(G \cap E^{\prime}\right)<\nu(G)+\epsilon \quad$ Hence $\nu(G \cap E)+\nu\left(G \cap E^{\prime}\right) \leq \nu(G)$ Consequently $E \in \mathcal{S}_{\nu}$ Thus $\nu$ has Property (*)

At the beginning of Section 3, it was mentioned that in [8] an example is given of a finite outer measure which although not regular, has Property (*).

We will present a family of finite outer measures which - regular or not - have Property $(*)$

PROPOSITION 3.10. Consider any lattice space $\langle X, \mathcal{L}\rangle$ and any element $M(\mathcal{L}), \mu$ Then $\mu^{\prime}$ has Property $(*)$.

PROOF. To show $\mu^{\prime}$ has Property $(*)$, consider any element of $\mathcal{P}(X), E$, such that $\mu^{\prime}(E)+$ $\mu^{\prime}\left(E^{\prime}\right)=\mu^{\prime}(X)$ and show $E \in \mathcal{S}_{\mu^{\prime}} \quad$ For this, use ([10], Thm 2.16), namely, $\mathcal{S}_{\mu^{\prime}}=$ $\left\{E \in \mathcal{P}(X) \mid \mu^{\prime}(E)=\sup \{\mu(L) \mid L \in \mathcal{L}\right.$ and $\left.L \subset E\}\right\}$. Note since $\mu^{\prime}(E)+\mu^{\prime}\left(E^{\prime}\right)=\mu^{\prime}(X)$ and $\mu^{\prime}(X)=\mu(X)<+\infty$,

$$
\begin{aligned}
\mu^{\prime}(E) & =\mu(X)-\mu^{\prime}\left(E^{\prime}\right) \\
& =\mu(X)-\inf \left\{\mu\left(L^{\prime}\right) \mid L^{\prime} \in \mathcal{L}^{\prime} \text { and } L^{\prime} \supset E^{\prime}\right\}, \text { by the definition of } \mu^{\prime}, \\
& =\sup \{\mu(L) \mid L \in \mathcal{L} \text { and } L \subset E\} .
\end{aligned}
$$

Then by the theorem mentioned above, $E \in \mathcal{S}_{\mu^{\prime}}$. Then $\mu^{\prime}$ has Property (*).

Observation. If $\mu \in I(\mathcal{L})$, then $\mu^{\prime}$ is regular.

COROLLARY 3.11. (1) $\mathcal{S}_{\mu_{\mathrm{r}}}=\left\{E \in \mathcal{P}(X) \mid \mu_{\imath}(E)=\mu^{\prime}(E)\right\}$. (2) $\mathcal{S}_{\mu_{\mathrm{r}}}=\mathcal{S}_{\mu^{\prime}}$

PROOF. Recall $\mu^{\prime}$ is a finitely subadditive outer measure (on $\mathcal{P}(X)$ ), $\mu^{\prime}$ is submodular, and for every element of $\mathcal{P}(X), E, \mu_{\imath}(E)=\mu(X)-\mu^{\prime}\left(E^{\prime}\right)$. Then by Prop 3.3, (1) $\mathcal{S}_{\mu}=\left\{E \in \mathcal{P}(X) \mid \mu_{\imath}(E)=\mu^{\prime}(E)\right\} ;$ (2) $\mathcal{S}_{\mu_{\mathrm{r}}}=\mathcal{S}_{\mu^{\prime}}$, since $\mu^{\prime}$ has Property (*) (by Prop 3.10).

PROPOSITION 3.12. If $\nu$ has Property (*), then (1) $\mathcal{S}_{\nu^{0}} \subset \mathcal{S}_{\nu^{\prime}}$ (2) $\nu^{0}$ has Property (*) (3) $\mathcal{S}_{\nu} \subset \mathcal{S}_{\nu^{0}}$. (4) $\mathcal{S}_{\nu}=\mathcal{S}_{\nu^{0}}$.

PROOF. Assume $\nu$ has Property (*)

(1) Consider any element of $\mathcal{S}_{\nu^{0}}, E$. Then 


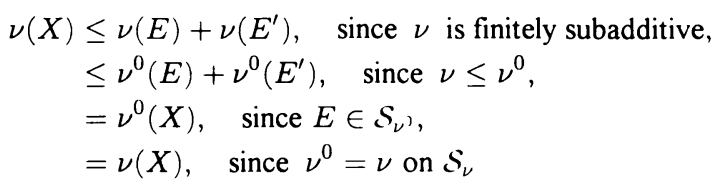

Hence $\nu(X)=\nu(E)+\nu\left(E^{\prime}\right)$ Hence since $\nu$ has Property (*) by assumption, $E \in \mathcal{S}_{\nu}$ Thus $\mathcal{S}_{\nu^{\circ}} \subset \mathcal{S}_{\nu}$

(2) To show $\nu^{0}$ has Property $(*)$, consider any element of $\mathcal{P}(X), E$, such that $\nu^{0}(E)+\nu^{0}\left(E^{\prime}\right)=\nu^{0}(X)$ and show $E \in \mathcal{S}_{\nu^{0}}$ Note to show $E \in \mathcal{S}_{\nu^{0}}$, it suffices to show for every element of $\mathcal{P}(X), G, \nu^{0}(G \cap E)+\nu^{0}\left(G \cap E^{\prime}\right) \leq \nu^{0}(G)$ Accordingly, consider any element of $\mathcal{P}(X)$, $G$. Now, consider any positive number $\epsilon$ Then since $\nu^{0}(G)=\inf \left\{\nu(S) \mid S \in \mathcal{S}_{\nu}\right.$ and $\left.S \supset G\right\}$ and $\nu^{0}(G)<+\infty$, there exists an element of $\mathcal{S}_{\nu}, S$, such that $S \supset G$ and $\nu(S)<\nu^{0}(G)+\epsilon$ Consider any such $S$ Then $\nu^{0}(G \cap E)+\nu^{0}\left(G \cap E^{\prime}\right) \leq \nu^{0}(S \cap E)+\nu^{0}\left(S \cap E^{\prime}\right)$, since $\nu^{0}$ is increasing Now, note $\nu(X) \leq \nu(E)+\nu\left(E^{\prime}\right) \leq \nu^{0}(E)+\nu^{0}\left(E^{\prime}\right)=\nu^{0}(X)$ (by assumption) $=\nu(X)$ Hence $\nu(X)=\nu(E)+$ $\nu\left(E^{\prime}\right) \quad$ Hence since $\nu$ has Property (*) by assumption, $E \in \mathcal{S}_{\nu}$ Consequently $\nu^{0}(G \cap E)+$ $\nu^{0}\left(G \cap E^{\prime}\right) \leq \nu^{0}(S \cap E)+\nu^{0}\left(S \cap E^{\prime}\right)=\nu(S \cap E)+\nu\left(S \cap E^{\prime}\right)=\nu(S)<\nu^{0}(G)+\epsilon$ Hence $\nu^{0}(G \cap E)+\nu^{0}\left(G \cap E^{\prime}\right) \leq \nu^{0}(G)$ Consequently $E \in \mathcal{S}_{\nu^{0}} \quad$ Thus $\nu^{0}$ has Property (*)

(3) Consider any element of $\mathcal{S}_{\nu}, E$. Now, note since $\nu^{0}$ has Property (*) by (2), to show $E \in \mathcal{S}_{\nu^{0}}$, it suffices to show $\nu^{0}(E)+\nu^{0}\left(E^{\prime}\right)=\nu^{0}(X)$ Note since $E \in \mathcal{S}_{\nu}, \nu^{0}(E)=\nu(E)$ and $\nu^{0}\left(E^{\prime}\right)=\nu\left(E^{\prime}\right)$ Then

$$
\begin{aligned}
\nu^{0}(E)+\nu^{0}\left(E^{\prime}\right) & =\nu(E)+\nu\left(E^{\prime}\right) \\
& =\nu(X), \quad \text { since } E \in \mathcal{S}_{\nu}, \\
& =\nu^{0}(X) .
\end{aligned}
$$

Hence $\nu^{0}(E)+\nu^{0}\left(E^{\prime}\right)=\nu^{0}(X)$. Consequently $E \in \mathcal{S}_{\nu^{0}}$. Thus $\mathcal{S}_{\nu} \subset \mathcal{S}_{\nu^{0}}$

PROPOSITION 3.13. (1) If $\nu$ has Property (*), then $\nu^{00}=\nu^{0}$, that is, $\nu^{0}$ is regular (2) If $\nu^{00}=\nu^{0}$, that is, if $\nu^{0}$ is regular, then $\nu^{0}$ has Property $(*)$.

PROOF. (1) Assume $\nu$ has Property (*). Now, to show $\nu^{00}=\nu^{0}$, consider any element of $\mathcal{P}(X), E$, and show $\nu^{00}(E)=\nu^{0}(E)$. Note $\nu^{00}(E)=\left(\nu^{0}\right)^{0}(E)=\inf \left\{\nu^{0}(S) \mid S \in \mathcal{S}_{\nu^{0}}\right.$ and $\left.S \supset E\right\}$, by definition Further, note since $\nu$ has Property (*) by assumption, $S_{\nu^{0}}=\mathcal{S}_{\nu}$ by (Prop 3 12, (4)) Consequently $\nu^{00}(E)=\inf \left\{\nu^{0}(S) \mid S \in \mathcal{S}_{\nu^{0}}\right.$ and $\left.S \supset E\right\}=\inf \left\{\nu(S) \mid S \in \mathcal{S}_{\nu}\right.$ and $\left.S \supset E\right\}=\nu^{0}(E)$, by the definition of $\nu^{0}$ Thus $\nu^{00}=\nu^{0}$.

PROPOSITION 3.14. (1) $\mathcal{S}_{\nu_{0}}=\left\{E \in \mathcal{P}(X) \mid \nu_{0}(E)=\nu^{0}(E)\right\}$. (2) $\mathcal{S}_{\nu^{0}} \subset \mathcal{S}_{\nu_{0}} \quad$ (3) $\mathcal{S}_{\nu^{0}}=\mathcal{S}_{\nu_{0}}$ iff $\nu^{0}$ has Property (*) (4) $\mathcal{S}_{\nu} \subset \mathcal{S}_{\nu_{0}}$. (5) If $\nu$ has Property (*), then $\mathcal{S}_{\nu^{0}}=\mathcal{S}_{\nu}=\mathcal{S}_{\nu_{0}}=$ $\left\{E \in \mathcal{P}(X) \mid \nu_{0}(E)=\nu^{0}(E)\right\}$.

PROOF. Note since $\nu^{0}$ is a finitely subadditive outer measure (on $\mathcal{P}(X)$ ), $\nu^{0}$ is submodular, and for every element of $\mathcal{P}(X), E, \nu_{0}(E)+\nu^{0}\left(E^{\prime}\right)=\nu(X)$ or, equivalently, $\nu_{0}(E)=\nu(X)-\nu^{0}\left(E^{\prime}\right)=$ $\nu^{0}(X)-\nu^{0}\left(E^{\prime}\right)$ by (Prop. 3.5, (4)), by Prop $33,(1),(2)$, and (3) are true

(4) Consider any element of $\mathcal{S}_{\nu}, E$. Then $\nu_{0}(E)=\nu(E)=\nu^{0}(E)$. Hence by (1), $E \in \mathcal{S}_{\nu_{0}}$. Thus $\mathcal{S}_{\nu} \subset \mathcal{S}_{\nu_{0}}$

(5) Assume $\nu$ has Property (*) Note $\mathcal{S}_{\nu^{0}}=\mathcal{S}_{\nu}$, by (Prop. 3.12, (4)) Further, note $\mathcal{S}_{\nu} \subset \mathcal{S}_{\nu^{0}}$ ((4) above). Also, note $\mathcal{S}_{\nu^{0}}=\mathcal{S}_{\nu_{0}}$, by [(Prop 312, (2)) and ((3) above)]. Then by ((1) above), $\mathcal{S}_{\nu^{0}}=\mathcal{S}_{\nu}=\mathcal{S}_{\nu_{0}}=\left\{E \in \mathcal{P}(X) \mid \nu_{0}(E)=\nu^{0}(E)\right\}$.

\section{Part (c)}

PROPOSITION 3.15. Consider any two finitely subadditive outer measures $\nu_{1}, \nu_{2}$ (on $\mathcal{P}(X)$ ), such that $\nu_{1} \leq \nu_{2}$ and $\nu_{1}(X)=\nu_{2}(X) \quad$ If $\nu_{1}$ has Property (*), then (1) $\mathcal{S}_{\nu_{1}} \supset \mathcal{S}_{\nu_{2}}$ $\nu_{20} \leq \nu_{10} \leq \nu_{1}^{0} \leq \nu_{2}^{0}$. (3) (2) implies (1)

PROOF. Assume $\nu_{1}$ has Property (*) 
(1) Consider any element of $\mathcal{S}_{\nu_{2}}, E$ Note to show $E \in \mathcal{S}_{\nu_{1}}$, since $\nu_{1}$ has Property (*), it suffices to show $\nu_{1}(E)+\nu_{1}\left(E^{\prime}\right)=\nu_{1}(X)$ Note

$$
\begin{aligned}
\nu_{1}(X) & \leq \nu_{1}(E)+\nu_{1}\left(E^{\prime}\right), \quad \text { since } \nu_{1} \text { is subadditive, } \\
& \leq \nu_{2}(E)+\nu_{2}\left(E^{\prime}\right), \quad \text { since } \nu_{1} \leq \nu_{2} \text { by assumption, } \\
& =\nu_{2}(X), \quad \text { since } E \in \mathcal{S}_{\nu_{2}} \\
& =\nu_{1}(X), \quad \text { by assumption }
\end{aligned}
$$

Hence $\nu_{1}(X)=\nu_{1}(E)+\nu_{1}\left(E^{\prime}\right) \quad$ Consequently $E \in \mathcal{S}_{\nu_{1}} \quad$ Thus $\mathcal{S}_{\nu_{1}} \supset \mathcal{S}_{\nu_{p}}$

(2) ( $\alpha$ ) Show $\nu_{20} \leq \nu_{10} \quad$ Consider any element of $\mathcal{P}(X), E$ Note $\nu_{20}(E)=\sup \left\{\nu_{2}(S) \mid S \in \mathcal{S}_{\nu_{2}}\right.$ and $S \subset E\}$ and $\nu_{10}(E)=\sup \left\{\nu_{1}(S) \mid S \in \mathcal{S}_{\nu_{1}}\right.$ and $\left.S \subset E\right\}$, by definition Now, consider any element of $\mathcal{S}_{\nu_{2}}, S$ Note

$$
\begin{aligned}
\nu_{2}(S) & =\nu_{2}(X)-\nu_{2}\left(S^{\prime}\right), \quad \text { since } S \in \mathcal{S}_{\nu_{2}} \\
& \leq \nu_{1}(X)-\nu_{1}\left(S^{\prime}\right), \quad \text { since } \nu_{1} \leq \nu_{2} \text { and } \nu_{1}(X)=\nu_{2}(X) \text { by assumption, } \\
& =\nu_{1}(S), \quad \text { since } S \in \mathcal{S}_{\nu_{1}} \text { by }(1)
\end{aligned}
$$

Consequently $\nu_{2}(S)=\nu_{1}(S) \quad$ Thus $\nu_{2}=\nu_{1}$ on $\mathcal{S}_{\nu_{2}} \quad$ Consequently $\nu_{20}(E)=\sup \left\{\nu_{2}(S) \mid S \in \mathcal{S}_{\nu_{2}}\right.$ and $S \subset E\} \leq \sup \left\{\nu_{1}(S) \mid S \in \mathcal{S}_{\nu_{1}}\right.$ and $\left.S \subset E\right\}=\nu_{10}(E)$ Thus $\nu_{20} \leq \nu_{10}$

( $\beta$ ) Show $\nu_{1}^{0} \leq \nu_{2}^{0} \quad$ Consider any element of $\mathcal{P}(X), E \quad$ Note $\nu_{1}^{0}(E)=\inf \left\{\nu_{1}(S) \mid S \in \mathcal{S}_{\nu_{1}}\right.$ and $S \supset E\}$ and $\nu_{2}^{0}(E)=\inf \left\{\nu_{2}(S) \mid S \in \mathcal{S}_{\nu_{2}}\right.$ and $\left.S \supset E\right\}$, by definition. Now, recall $\nu_{2}=\nu_{1}$ on $\mathcal{S}_{\nu_{2}}$ (from part $(\alpha)$ ) Consequently $\nu_{1}^{0}(E)=\inf \left\{\nu_{1}(S) \mid S \in \mathcal{S}_{\nu_{1}} \quad\right.$ and $\left.S \supset E\right\} \leq \inf \left\{\nu_{2}(S) \mid S \in \mathcal{S}_{\nu_{2}} \quad\right.$ and $S \supset E\}=\nu_{2}^{0}(E)$ Thus $\nu_{1}^{0} \leq \nu_{2}^{0}$

( $\gamma$ ) Consequently $\nu_{20} \leq \nu_{10} \leq \nu_{1}^{0} \leq \nu_{2}^{0}$

(3) Assume (2) and show (1) Note $\nu_{20}=\nu_{2}^{0}$ on $\mathcal{S}_{\nu_{2}}$ Then $\nu_{10}=\nu_{1}^{0}$ on $\mathcal{S}_{\nu_{2}}$, using the assumption. Hence $\mathcal{S}_{\nu_{2}} \subset \mathcal{S}_{\nu_{1} 0}$, by (Prop. 314 , (1)) Further, note since $\nu_{1}$ has Property (*) by assumption, $\mathcal{S}_{\nu_{10}} \subset \mathcal{S}_{\nu_{1}}$, by (Prop 3.14, (5)). Consequently $\mathcal{S}_{\nu_{1}} \supset \mathcal{S}_{\nu_{2}}$

\section{APPLICATIONS OF PROPOSITION 3.15.}

APPLICATION 3.16. Consider any two elements of $M(\mathcal{L}), \mu, \nu$, such that $\mu \leq \nu$ and $\mu(X)=\nu(X)$. Further, consider $\mu^{\prime}, \nu^{\prime} \quad$ Recall the facts: $\mu^{\prime}$ and $\nu^{\prime}$ are finitely subadditive outer measures (on $\mathcal{P}(X)) ; \nu^{\prime} \leq \mu^{\prime}$ and $\nu^{\prime}(X)=\mu^{\prime}(X) ; \nu^{\prime}$ has Property (*) (Prop. 3.10). Hence by (Prop. $3.15,(1)), \mathcal{S}_{\nu^{\prime}} \supset \mathcal{S}_{\mu^{\prime}}$.

APPLICATION 3.17. Consider any element of $M(\mathcal{L}), \mu$. Further, consider $\mu^{\prime}, \mu^{\prime \prime}$ Recall the facts $\mu^{\prime}$ is a finitely subadditive outer measure (on $\mathcal{P}(X)$ ) and $\mu^{\prime \prime}$ is a finitely subadditive outer measure (on $\mathcal{P}(X)$ ); $\mu^{\prime \prime} \leq \mu^{\prime}$; if $\mu \in M_{\sigma}(\mathcal{L})$, then $\mu^{\prime \prime}(X)=\mu^{\prime}(X)$ (see [10]) Consequently, according to (Prop. 315 , (1)), if $\mu \in M_{\sigma}(\mathcal{L})$ and $\mu^{\prime \prime}$ has property $(*)$, then $\mathcal{S}_{\mu^{\prime \prime}} \supset \mathcal{S}_{\mu^{\prime}}$. Finally, recall the fact. If a finitely subadditive outer measure (on $\mathcal{P}(X)$ ) is regular, then it has Property (*) (Prop. 39 ). Consequently if $\mu \in M_{\sigma}(\mathcal{L})$ and $\mu^{\prime \prime}$ is regular, then $\mathcal{S}_{\mu^{\prime \prime}} \supset \mathcal{S}_{\mu^{\prime}}$.

APPLICATION 3.18. Consider any element of $M^{\sigma}(\mathcal{L})$. Further, consider $\mu^{\prime}, \mu^{\prime \prime}$, and $\mu^{*}$ (where $\mu^{*}$ is the outer measure induced by $\mu$ ). Recall the facts: $\mu^{\prime}, \mu^{\prime \prime}$, and $\mu^{*}$ are finitely subadditive outer measures (on $\mathcal{P}(X)$ ), $\mu^{*} \leq \mu^{\prime \prime} \leq \mu^{\prime}$ (hence $\mu^{*}(X)=\mu^{\prime \prime}(X)=\mu^{\prime}(X)$ ); $\mu^{*}$ is regular (hence $\mu^{*}$ has Property (*)). Hence by (Prop 3.15, (1)), $\mathcal{S}_{\mu^{*}} \supset \mathcal{S}_{\mu^{\prime}}$ and $\mathcal{S}_{\mu^{*}} \supset \mathcal{S}_{\mu^{\prime \prime}}$. Further, recall the fact If, in addition, $\mu$ is $\mathcal{L}$-regular, then $\mu^{*}=\mu^{\prime \prime}$. (Consequently $\mu^{\prime \prime}$ is regular.) Consequently if $\mu \in M_{R}^{\sigma}(\mathcal{L})$, then $\mathcal{S}_{\mu^{*}}=\mathcal{S}_{\mu^{*}} \supset \mathcal{S}_{\mu^{\prime}}$

ACKNOWLEDGMENT. The author wishes to express his appreciation to Long Island University for partial support of the present work through a grant of released time from teaching duties. 


\section{REFERENCES}

[1] ALEXANDROFF, A D ,. Additive set-functions in abstract spaces, Mat. Sb. (N.S.) 9 (51) (1941), 563-628

[2] BACHMAN, G and SULTAN, A, On regular extensions of measures, Pacıfic J. Math., 86 (1980), 389-395

[3] CAMACHO, J, On maximal measures with respect to a lattice, Internat. J. Math. \& Math. Sct., 14 (1991), 93-98

[4] EID, G, On normal lattices and Wallman spaces, Internat. J. Math. \& Math. Scl., 13 (1990), 3138

[5] FROLIK, Z, Prime filters with the c i p , Comm. Math. Univ. Carolinae, 13 (1972), 553-575

[6] KINDLER, J , Supermodular and tight set-functions, Math. Nachr., 134 (1987), 131-147

[7] LEMBCKE, J, Konservative Abbildungen und Fortesung regularer Masse, Z. Wahr. 15 (1970), 57-96

[8] MUNROE, M, Introduction to Measure and Integratıon, Addison-Wesley, Cambridge, MA (1953).

[9] STRATIGOS, P, Lattice separation and outer measures, J. Math. Anal. and Appl., 166 (1992), 84-102

[10] STRATIGOS, P., Lattice measures and associated outer measures, Period. Math. Hungar., 27 (1993), 35-56

[11] SZETO, M, On maximal measures with respect to a lattice, Measure Theory and Its Applications, Proceedings of the 1980 Conference, Northern Illinois University, 1980. 


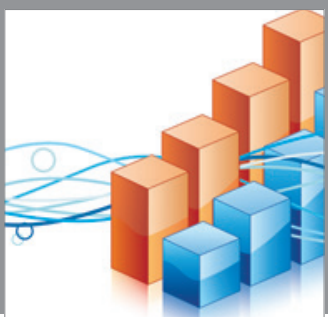

Advances in

Operations Research

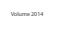

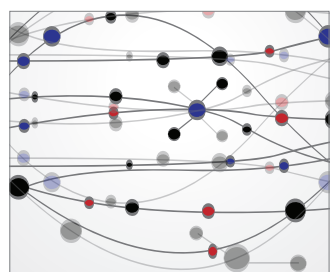

\section{The Scientific} World Journal
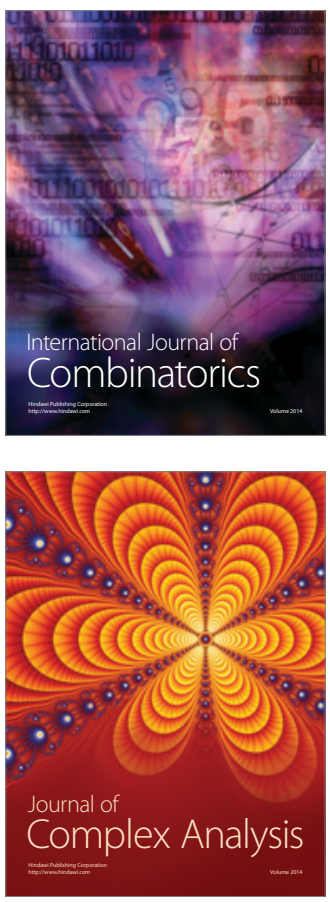

International Journal of

Mathematics and

Mathematical

Sciences
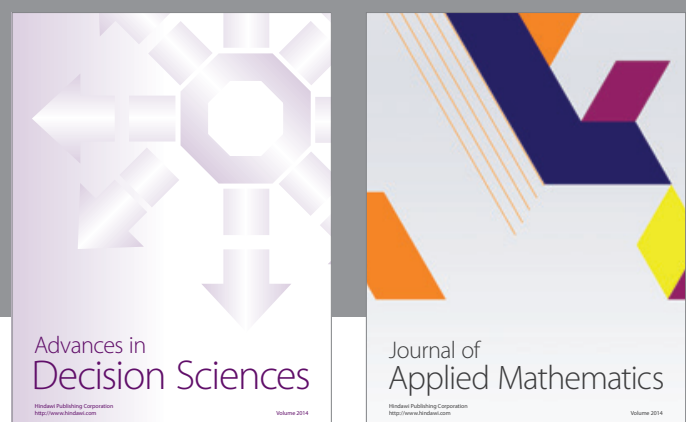

Journal of

Applied Mathematics
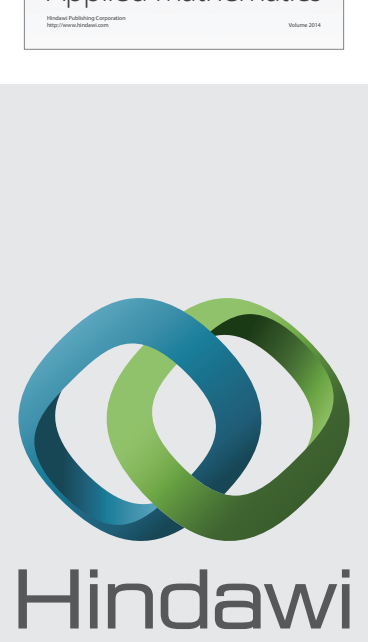

Submit your manuscripts at http://www.hindawi.com
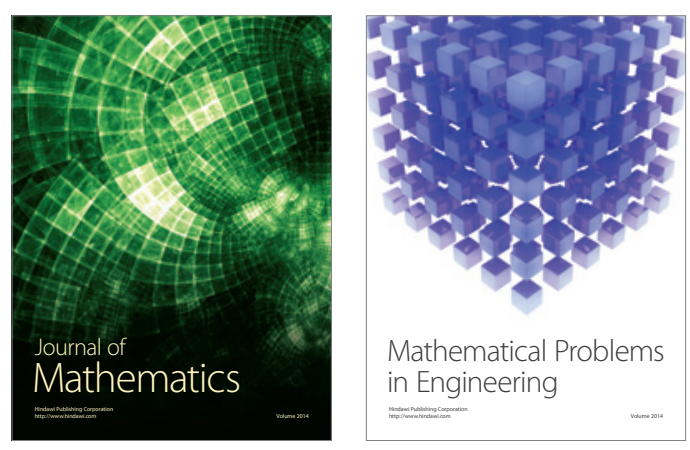

Mathematical Problems in Engineering
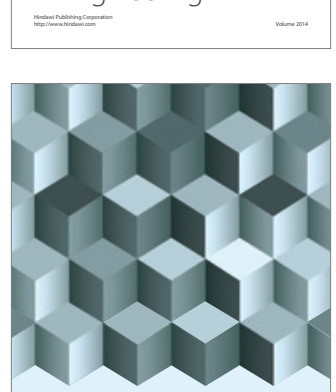

Journal of

Function Spaces
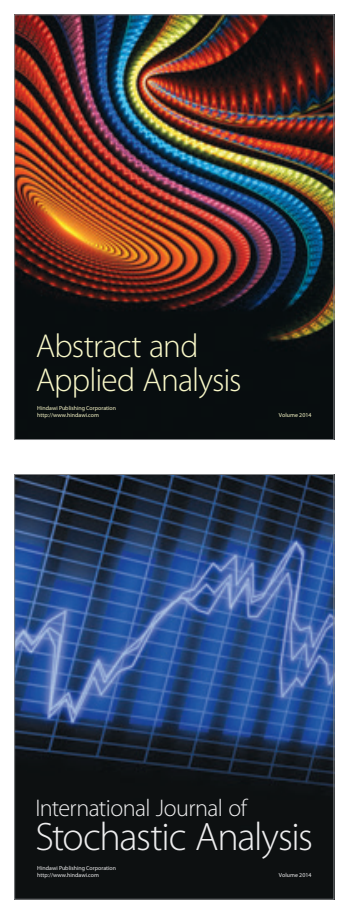

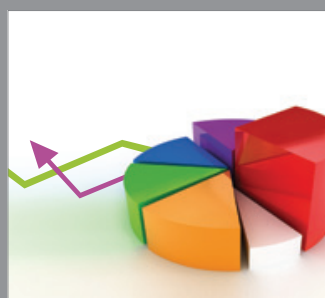

ournal of

Probability and Statistics

Promensencen
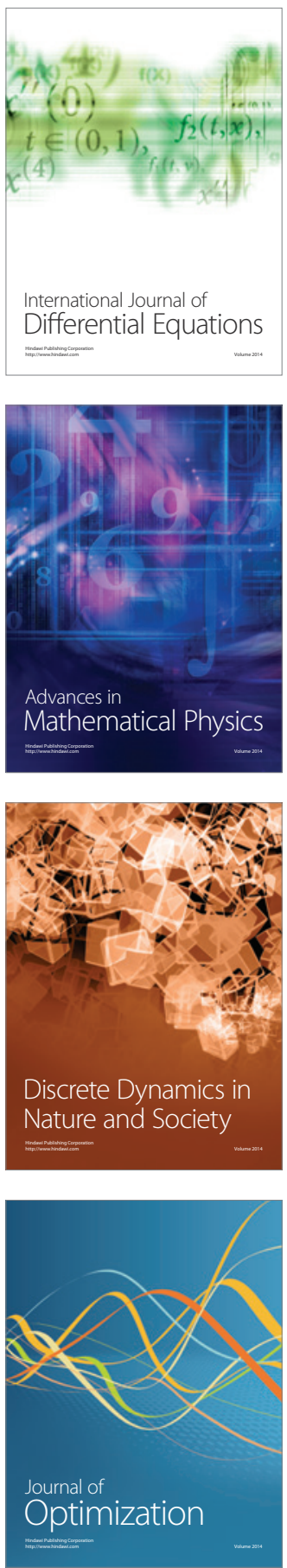